\title{
A Multicenter Study of a Fluorescence In Situ Hybridization Probe Set for Diagnosing High-Grade Dysplasia and Adenocarcinoma in Barrett's Esophagus
}

John M. Poneros ${ }^{1}$, Adam S. Faye ${ }^{2}$, Emily G. Barr Fritcher ${ }^{3}$, Ananda Sen $^{4,14}$, Sharmila Anandasabapathy ${ }^{5}$, Robert S. Bresalier ${ }^{6}$, Norman Marcon ${ }^{7,15}$, D. Kim Turgeon ${ }^{8,16}$, Henry Appelman $^{9}$, Daniel Normolle ${ }^{10}$, Larry E. Morrison ${ }^{11,13}$, Dean E. Brenner ${ }^{8,12}$, and Kevin C. Halling ${ }^{3}$

${ }^{1}$ Department of Gastroenterology, Columbia University, Medical Center, 161 Fort Washington Avenue, Suite 862,, New York, NY 10032, USA

${ }^{2}$ Department of Internal Medicine, Columbia University, Medical Center, 177 Fort Washington Avenue, Milstein 6C-, 12, New York, NY 10032, USA

${ }^{3}$ Department of Laboratory Medicine and Pathology, Mayo, Clinic, 200 First SW, Rochester, MN 55905, USA

${ }^{4}$ Department of Biostatistics, University of Michigan Medical, Center, Ann Arbor, MI, USA

${ }^{5}$ Department of Gastroenterology, Baylor College of, Medicine, Baylor St. Luke's Medical Center Clinic, 7200, Cambridge Street Suite 10C, Houston, TX 77030, USA

${ }^{6}$ Department of Gastroenterology, University of Texas M.D., Anderson Cancer Center, 1515

Holcombe Blvd, Unit 1466, Houston, TX 77030, USA

${ }^{7}$ Department of Gastroenterology, University of Toronto,, Toronto, ON, Canada

${ }^{8}$ Department of Internal Medicine, University of Michigan, Medical Center, Ann Arbor, MI, USA

${ }^{9}$ Department of Pathology, University of Michigan Medical, Center, 1301 Catherine St., Ann Arbor, MI 48109, USA

${ }^{10}$ Department of Biostatistics, University of Pittsburgh Cancer, Institute, 201 North Craig Street, Sterling Plaza Suite 325,, Pittsburgh, PA 15213, USA

${ }^{11}$ Abbott Molecular, Inc., Des Plaines, IL, USA

${ }^{12}$ Department of Pharmacology, University of Michigan, Medical Center, 1500 E Medical Center Dr \#2150,, Ann Arbor, MI 48109, USA

\footnotetext{
Correspondence to: John M. Poneros.

${ }^{13}$ Present Address: Ventana Medical Systems Inc., 1910 E., Innovation Park Dr., Tucson, AZ 85755, USA

Compliance with ethical standards

Conflict of interest Dr. Larry Morrison was an employee of Abbott Molecular, Inc. at the time this work was performed, and owns stocks and options to purchase stock from Abbott and AbbVie. Dr. Larry Morrison currently works at Ventana Medical Systems Inc., a diagnostics company in the Roche Group, and owns stocks and options to purchase stock in the Roche Group. Dr. Kevin Halling is the co-inventor of the FISH probe set discussed in this paper, receives royalties from its sale, and also receives Grant Funding from Abbott Molecular Inc.
} 
${ }^{14}$ Department of Family Medicine, University of Michigan, Medical School, 1018 Fuller St., Ann Arbor, MI 48104, USA

${ }^{15}$ St. Michael's Hospital, University of Toronto, 30 Bond, Street, 16-062 Cardinal Carter South Wing, Toronto,, ON M5B 1W8, Canada

${ }^{16}$ Department of Gastroenterology, University of Michigan, Health System, Taubman Center Floor 3, Reception D,, 1500 E Medical Center Dr, Ann Arbor, MI 48109, USA

\section{Abstract}

Background and Aims-Preliminary single-institution data suggest that fluorescence in situ hybridization (FISH) may be useful for detecting high-grade dysplasia (HGD) and esophageal adenocarcinoma (EA) in patients with Barrett's esophagus (BE). This multicenter study aims to validate the measurement of polysomy (gain of at least two loci) by FISH as a way to discriminate degrees of dysplasia in BE specimens.

Methods-Tissue specimens were collected from four different hospitals and read by both the local pathology department ("Site diagnosis") and a single central pathologist ("Review diagnosis") at a separate institution. The specimens then underwent FISH analysis using probes 8q24 (MYC), 9p21 (CDKN2A), 17q12 (ERBB2), and 20q13 (ZNF217) for comparison. A total of 46 non-BE, 42 non-dysplastic specialized intestinal metaplasia (SIM), 23 indefinite-grade dysplasia (IGD), 10 low-grade dysplasia (LGD), 29 HGD, and 42 EA specimens were analyzed.

Results-We found that polysomy, as detected by FISH, was the predominant chromosomal abnormality present as dysplasia increased. Polysomy was also the best predictor for the presence of dysplasia or EA when comparing its area under the curve to that of other FISH abnormalities. We observed that if at least $10 \%$ of cells had polysomy within a specimen, the FISH probe was able to differentiate between EA/HGD and the remaining pathologies with a sensitivity of $80 \%$ and a specificity of $88 \%$.

Conclusions-This study demonstrates that using FISH to determine the percentage of cells with polysomy can accurately and objectively aid in the diagnosis of HGD/EA in BE specimens.

\section{Keywords}

Barrett's esophagus; Dysplasia; Histopathology; Endoscopy; FISH; Polysomy

\section{Introduction}

Barrett's esophagus is a preneoplastic condition in which the lining of the distal esophagus changes from normal squamous epithelium to SIM, a precursor for progression to EA. The incidence of EA has increased over the past few decades, with prognosis linked to the stage of the tumor [1-4]. Individuals diagnosed in the early stages of EA can have 5-year survival rates as high as $95 \%$, whereas those diagnosed with more advanced disease have only a 10$15 \%$ 5-year survival rate [5]. As a result, major emphasis has been placed upon the early detection of dysplasia and EA in order to eradicate these high-grade lesions and improve overall survival. 
The current standard of practice is periodic four-quadrant surveillance biopsies in each centimeter of the involved esophageal segment if dysplasia is present. This allows for stratification of individuals based upon degree of dysplasia and subsequently helps determine likelihood of progressing to EA. However, adherence to these guidelines appears to be low, thus limiting the detection of high-grade dysplastic lesions [6]. Additionally, this method is limited in two substantial ways: (1) sampling error and (2) high pathologist interobserver variability [7]. Sampling error can occur in tissue collection because early or subtle mucosal changes in dysplastic tissue may be patchy, and offer little macroscopic evidence of its premalignant potential [8]. After collection, diagnosis by a pathologist can have significant variability. This is most pronounced in distinguishing between the lower-grade dysplastic lesions, but notably only approximately $85 \%$ inter-observer agreement is measured when diagnosing high-grade dysplasia and EA versus all other forms of dysplasia (including no dysplasia) $[9,10]$. Thus, improved more objective methods of risk stratification for BE patients are needed.

Several studies demonstrate that an increasing number of genetic alterations occur as SIM progresses to EA, and exploration of these markers to diagnose dysplasia is an active area of research [11-13]. In 2006, FISH probes comprising four loci, 8q24 (MYC), 9p21

(CDKN2A), 17q11.2 (ERBB2), and 20q13.2 (ZNF217), showed high sensitivity and specificity for identifying HGD and EA, and this method was shown to be superior to digital image analysis (DIA) and cytology [14, 15]. Furthermore, polysomy, as detected by FISH, was the most commonly detected abnormality in areas of dysplasia and EA, and the most likely predictor of progression to HGD/EA $[15,16]$. This multicenter study aims to validate the measurement of polysomy by FISH as a way to objectively discriminate degrees of dysplasia of BE biopsy specimens.

\section{Methods}

\section{Clinical Ascertainment}

Forty-five patients with known BE and no prior eradication therapy were enrolled at four different institutions: University of Michigan Health System, Brigham and Women's Hospital, University of Texas MD Anderson Cancer Center, and St. Michael's Hospital in Toronto. BE tissue specimens were collected during standard of care surveillance endoscopies. A random subset of the specimens collected from each consented patient were then used for analysis. A "Site diagnosis" was read by that institution's pathology department. The "Review diagnosis" was determined by re-sectioning the paraffinembedded tissue and having these slides read by a single gastrointestinal pathologist at the University of Michigan Health System ("central pathologist"). This central pathologist was blinded to the Site diagnosis read. The same slides read by the central pathologist were then sent to the Mayo Clinic for FISH analysis. The potential diagnoses in order of increasing severity were: no evidence of BE (normal cardia and squamous epithelium), SIM (specialized intestinal metaplasia), IGD (indefinite-grade dysplasia), LGD (low-grade dysplasia), HGD (high-grade dysplasia), and EA (esophageal adenocarcinoma). Data were analyzed using the Site diagnosis, the Review diagnosis, and the highest grade diagnosis within the entire BE segment of each patient (" Overall diagnosis"). The Overall diagnosis 
is clinically applicable because the patient's management is determined by the highest grade lesion within the esophagus. No baseline demographic data were collected as all tissue specimens were subsequently de-identified for analysis. A total of 46 non-BE, 42 SIM, 23 IGD, 10 LGD, 29 HGD, and 42 EA specimens were analyzed. The institutional review boards of each participating institution approved this study.

FISH

Unstained formalin-fixed paraffin-embedded slides were pretreated and hybridized with probes provided by Abbott Molecular (DesPlaines, IL) directed to 8q24 (MYC), 9p21 (CDKN2A), 17q11.2 (ERBB2), and 20q13.2 (ZNF217), as previously described [14]. FISH signal patterns for 50 nuclei within the histologic area of interest were enumerated by a technologist experienced in FISH analysis. Each cell analyzed was categorized as FISH negative (having the anticipated number of two FISH signals per probe) or FISH abnormal (having more or less than the anticipated number of FISH signals). Gain of a locus was defined as a single locus having greater than two signals, and loss of a locus was defined a single locus having less than two signals. FISH abnormality types included polysomy (gain of at least two loci), single locus gain (3-9 signals of a single locus and two copies of other loci), amplification of a single locus ( $\geq 10$ signals of a single locus and two copies of other loci), and 9p21 loss (0-1 signals of 9p21 and two copies of other loci). The predominant FISH abnormality was defined as the most common abnormality (i.e., highest percentage) detected within a specimen.

\section{Statistical Analysis}

Statistical analyses were performed with SAS version 9.4 and IBM SPSS version 22 . The kappa statistic was calculated as a measure of agreement between Site diagnosis and Review diagnosis for varying degrees of dysplasia. Receiver operator curves were used in order to compare different FISH chromosomal abnormalities as diagnostic criteria for determining degree of dysplasia. In order to determine the best predictor of dysplasia, the area under the receiver operator curves (AUCs) for various FISH abnormality types were plotted using a bootstrap analysis. $P$ values were then computed using the method appropriate for comparing correlated AUCs [17]. Given that prior single-institution data found polysomy to be the most commonly detected abnormality in areas of dysplasia and EA, we focused our analysis on the validation of polysomy as a sensitive and specific marker for detecting varying degrees of dysplasia and EA.

\section{Results}

A total of 192 specimens were analyzed by a site pathologist, central pathologist, and FISH analysis using probes directed to 8q24 (MYC), 9p21 (CDKN2A), 17q11.2 (ERBB2), and 20q13.2 (ZNF217). The histologic breakdown of the specimens as recorded by the central pathologist was 46 non-BE, 42 SIM, 23 IGD, 10 LGD, 29 HGD, and 42 EA specimens. Inter-observer agreement was calculated between the central and site pathologists. The kappa statistic for agreement on identification of EA/HGD versus rest was .79, with a value of 1.0 indicating $100 \%$ agreement between the different reads. In accordance with 
previously published studies, higher inter-observer agreement was seen when distinguishing EA and HGD from other degrees of dysplasia (Table 1).

To validate FISH polysomy as a marker for detection of dysplasia in these specimens, the area under the receiver operating curves were calculated to compare different FISH abnormalities as predictors for the central pathologist's Review diagnosis, our gold standard. Table 2 shows the AUC values for both cells with polysomy and cells with 9p21 loss as the predominant FISH abnormality, and the comparative $p$ values. Previous studies used positive criteria for FISH to include polysomy, gain of a single locus and 9p21 loss [13]. In our data, polysomy had the highest discriminatory power for most comparisons, with an AUC of .83 in discriminating EA/HGD from all other diagnoses. Interestingly, similar values were seen when looking at the AUC for percentage of cells with polysomy as a predictor of both the Site diagnosis and Overall diagnosis. Of note, adding SIM to the comparison causes 9p21 loss to become the predominant FISH abnormality, which lessens the AUC for polysomy. The AUC for the percentage of cells with a predominant 9p21 loss for EA/HGD/LGD/IGD/SIM versus rest was .90, whereas the AUC for the percentage of cells with polysomy for the same comparison was .76. An additional analysis that included any FISH abnormality (polysomy, single gain, amplification, and 9p21 loss) resulted in an AUC of .75 for HGD/EA samples versus rest.

Although many types of chromosomal abnormalities are present for each respective histologic classification, polysomy is most commonly observed with increasing degrees of dysplasia (Fig. 1). Furthermore, as SIM progresses to EA, the percent of specimens with polysomy as the most common FISH abnormality also increased from approximately 5 to $80 \%$, respectively. Of note, all 46 specimens that were negative for BE had no cells with polysomy.

We examined the receiver operator curve for polysomy to select an optimal cutoff value of $10 \%$. When compared to the Overall diagnosis, if at least $10 \%$ cells in a specimen were positive for polysomy, we were able to obtain a sensitivity and specificity of 88 and $75 \%$ for EA versus rest and a sensitivity and specificity of 80 and $88 \%$ for EA/HGD versus rest, respectively. When LGD and IGD were added, specificity increased to $96 \%$ but sensitivity decreased to $74 \%$ (Table 3).

Since early or subtle mucosal changes in dysplastic tissue may be small and frequently not identifiable by gross examination, we explored whether high-grade lesions elsewhere in the esophagus could lead to a higher number of cells having amplified loci in the surrounding non-HGD/ EA tissue, a so-called field effect. From our analyses, we found 7/7 (100\%) of IGD/LGD specimens to have evidence of amplification or polysomy when HGD was present elsewhere in the same esophageal segment (Table 4).

\section{Discussion}

Current methods of monitoring BE patients for progression to EA rely on random sampling of mucosa and have high inter-observer variability among pathologists [7]. Several studies have examined alternative ways to measure degree of dysplasia, specifically comparing 
FISH to both DIA (digital image analysis) and cytology [15]. Using a four-probe combination developed in 2006, FISH was found to have superior sensitivity for determining LGD, HGD, and EA as compared to DIA and cytology. Since then, studies have used different criteria for FISH positivity and have focused upon revealing the cytogenetic changes as normal mucosa progresses to EA [14-16, 18, 19]. Polysomy is the most commonly detected abnormality in areas of dysplasia and EA, and our study focused on using polysomy by FISH to analyze the degree of dysplasia in order to limit pathologist inter-observer variability and validate a more objective measure of dysplasia [15].

This multicenter study builds upon single-institution data that suggest that FISH may be useful for the detection of dysplasia and EA in patients with BE. In concordance with previous findings, our study demonstrated an increasing number of specimens with polysomy as the predominant chromosomal abnormality detected by FISH as the degree of dysplasia increased (Fig. 1). This indicates that cellular progression toward adenocarcinoma coincides with chromosomal aberrations at these probe sites resulting in gain of loci. This is true with the exception of the $9 \mathrm{p} 21$ locus, which is the site of a tumor suppressor gene $(C D K N 2 A)$ that is usually lost as the cell progresses from normal mucosa to $\mathrm{BE}[16,20]$.

In comparing the AUC for each FISH abnormality, we focused on 9p21 loss, since it is an early and pervasive alteration in the change to metaplasia. Using the Review diagnosis, we found the AUC for polysomy to be superior for most histologic comparisons (Table 2). Similar AUC values were noted when using the Overall diagnosis, indicating that the percentage of cells with polysomy was able to predict the patient's highest grade pathologic diagnosis with comparable accuracy. Of note, for both the Review and Overall diagnosis, the AUC for the percentage of cells with 9p21 loss as the predominant abnormality for $\mathrm{EA} / \mathrm{HGD} / \mathrm{LGD} / \mathrm{IGD} / \mathrm{SIM}$ versus rest was greater than the AUC for the percentage of cells with polysomy for the same histologic comparison. We expect this result because 9p21 deletion is an early marker often found in metaplasia, whereas polysomy is more prevalent in dysplasia and EA.

We found that polysomy by FISH had excellent diagnostic capabilities in discriminating HGD/EA from the remaining histologic diagnoses, a clinically important distinction, as these diagnoses frequently lead to endoscopic treatment and oncologic evaluation. As a result, FISH may potentially be utilized in conjunction with pathology, as current guidelines state that any diagnosis of dysplasia must be confirmed. Traditionally, this has resulted in a second pathologist with expertise in BE reviewing the specimen. However, given the high diagnostic accuracy of FISH in detecting HGD/EA, FISH analysis may be used to confirm pathologic diagnoses of HGD and obviate the need for a second pathologist's review. When LGD and IGD were added, specificity greatly increased at the expense of sensitivity. In a screening setting, a high sensitivity would be preferable to maximize the number of cases identified. Of note, our results demonstrate improved sensitivity and specificity in detecting HGD/EA as compared to cytology (both brush and balloon), and digital image analysis [15, 21].

Our study also suggests the presence of a "field effect," potentially enhancing the use of FISH as a screening tool during endoscopy. Our data, although limited in power, suggest that 
genetic changes may occur in nearby tissue when HGD is present elsewhere in the segment. More specifically, we found that all esophageal segments read as IGD/LGD had evidence of amplification or polysomy only when HGD was found elsewhere in the patient's esophageal column. Additionally, recent evidence has suggested that FISH may play a role in riskstratifying individuals with dysplasia. A retrospective analysis of patients with HGD found that if more than 4 of 100 cells had evidence of polysomy, there was a higher likelihood of progression to EA [22]. Although further verification is needed, these findings suggest that genetic changes detected by FISH can both predict HGD lesions elsewhere in the esophageal column as well as progression to EA. As a screening tool, this has the potential to reduce the effect of sampling bias by limiting the number of HGD lesions missed, as well as guide future management through further risk stratification of patients with dysplasia.

One potential factor limiting the widespread use of FISH is its availability. In practice, biopsies taken during a surveillance endoscopy would first be read by an experienced pathologist, re-sectioned, and then sent for FISH analysis. Currently, although many commercial laboratories and academic centers have the necessary equipment and technologists required to run FISH, it is not widely available. Analogous to sending a specimen to a center with an experienced pathologist, the tissue would need to be sent to a center with FISH capabilities. Although the FISH results would be ready in only a few days, sending the specimens for processing would incur an additional cost that is on the order of a few hundred dollars.

One limitation of our study is that our kappa statistic appears to be higher than those previously reported [10]. In our analysis, we focused on binary comparisons, and we evaluated agreement between two specialized pathologists. This may inflate our observed kappa statistic. Additionally, it should be noted that re-sectioning tissue may contribute to differences in the Site and Review diagnoses, although this is likely negligible given that only a few microns were used to cut each tissue block.

In conclusion, this study demonstrated a high detection rate for HGD and EA using a FISH panel with probes to 8q24 (MYC), 9p21 (CDKN2A), 17q12 (ERBB2), and 20q13 (ZNF217). Additionally, FISH analysis of LGD/IGD specimens was able to accurately predict the presence of high-grade dysplastic lesions elsewhere within the patient's BE segment. Given its high objective diagnostic accuracy for identifying HGD/EA and potential to detect higher-grade histology at other levels in the BE segment, FISH may be useful in conjunction with traditional histologic analysis, both as a diagnostic marker and screening tool to identify those at higher risk of progression to EA. Future prospective studies specifically examining its use as an adjunctive marker are needed in order to further investigate its promising and clinically relevant potential.

\section{Acknowledgments}

This study was supported by the Grants DN: NIH/NCI P30CA047904, DB: EDRN CA86400.

\section{Abbreviations}

FISH Fluorescence in situ hybridization 
HGD High-grade dysplasia

EA Esophageal adenocarcinoma

BE Barrett's esophagus

SIM Specialized intestinal metaplasia

IGD Indefinite-grade dysplasia

LGD Low-grade dysplasia

AUC Area under the receiver operator curve

\section{References}

1. Devesa SS, Blot WJ, Fraumeni JF Jr. Changing patterns in the incidence of esophageal and gastric carcinoma in the United States. Cancer. 1998; 83:2049-2053. [PubMed: 9827707]

2. Polednak AP. Trends in survival for both histologic types of esophageal cancer in US surveillance, epidemiology and end results areas. Int J Cancer. 2003; 105:98-100. [PubMed: 12672037]

3. Pohl H, Welch HG. The role of overdiagnosis and reclassification in the marked increase of esophageal adenocarcinoma incidence. J Natl Cancer Inst. 2005; 97:142-146. [PubMed: 15657344]

4. Zhang Y. Epidemiology of esophageal cancer. World J Gastroenterol. 2013; 19:5598-5606. [PubMed: 24039351]

5. Enzinger PC, Mayer RJ. Esophageal cancer. N Engl J Med. 2003; 349:2241-2252. [PubMed: 14657432]

6. Abrams JA, Kapel RC, Lindberg GM, et al. Adherence to Biopsy Guidelines for Barrett's Esophagus Surveillance in the Community Setting in the United States. Clin Gastroenterol Hepatol. 2009; 7:736-742. [PubMed: 19268726]

7. Spechler SJ. Dysplasia in Barrett's esophagus: limitations of current management strategies. Am J Gastroenterol. 2005; 100:927-935. [PubMed: 15784042]

8. Reid BJ, Weinstein WM, Lewin KJ, et al. Endoscopic biopsy can detect high-grade dysplasia or early adenocarcinoma in Barrett's esophagus without grossly recognizable neoplastic lesions. Gastroenterology. 1988; 94:81-90. [PubMed: 3335302]

9. Reid BJ, Haggitt RC, Rubin CE. Observer variation in the diagnosis of dysplasia in Barrett's esophagus. Hum Pathol. 1988; 19:166-178. [PubMed: 3343032]

10. Montgomery E, Bronner MP, Goldblum JR, et al. Reproducibility of the diagnosis of dysplasia in Barrett esophagus: a reaffirmation. Hum Pathol. 2001; 32:368-378. [PubMed: 11331953]

11. Riegman PH, Vissers KJ, Alers JC, et al. Genomic alterations in malignant transformation of Barrett's esophagus. Cancer Res. 2001; 61:3164-3170. [PubMed: 11306503]

12. Galipeau PC, Prevo LJ, Sanchez CA, et al. Clonal expansion and loss of heterozygosity at chromosomes 9p and 17p in premalignant esophageal (Barrett's) tissue. J Natl Cancer Inst. 1999; 91:2087-2095. [PubMed: 10601379]

13. Falk GW, Skacel M, Gramlich TL, et al. Flourescence in situ hybridization of cytologic specimens from Barrett's esophagus: a pilot feasibility study. Gastroint Endosc. 2004; 60:280-284.

14. Brankley SM, Wang KK, Harwood AR, et al. The development of a fluorescence in situ hybridization assay for the detection of dysplasia and adenocarcinoma in Barrett's esophagus. J Mol Diagn. 2006; 8:260-267. [PubMed: 16645214]

15. Fritcher EG, Brankley SM, Kipp BR, et al. A comparison of conventional cytology, DNA ploidy analysis, and fluorescence in situ hybridization for the detection of dysplasia and adenocarcinoma in patients with Barrett's esophagus. Hum Pathol. 2008; 39:1128-1135. [PubMed: 18602665]

16. Brankley SM, Fritcher EG, Smyrk TC, et al. Fluorescence in situ hybridization mapping of esophagectomy specimens from patients with Barrett's esophagus with high grade dysplasia or adenocarcinoma. Hum Pathol. 2012; 43:172-179. [PubMed: 21820152] 
17. DeLong ER, DeLong DM, Clarke-Pearson DL. Comparing the areas under two or more correlated receiver operating characteristic curves: a nonparametric approach. Biometrics. 1988; 44:837-845. [PubMed: 3203132]

18. Rossi E, Villanacci V, Basotti G, et al. TOPOIIalpha and HER-2/ neu overexpression/amplification in Barrett's oesophagus, dysplasia and adenocarcinoma. Histopathology. 2010; 57:81-89. [PubMed: 20557373]

19. Rossi E, Grisanti S, Villanacci V, et al. HER-2 overexpression/ amplification in Barrett's esophagus predicts early transition from dysplasia to adenocarcinoma: a clinico-pathologic study. J Cell Mol Med. 2009; 9B:3826-33.

20. Sanz-Ortega J, Hernández S, Saez MC, et al. 3p21, 5q21, 9p21 and 17p13.1 allelic deletions are potential markers of individuals with a high risk of developing adenocarcinoma in Barrett's epithelium without dysplasia. Hepatogastroenterology. 2003; 50:404-407. [PubMed: 12749233]

21. Sami SS, Ragunath K, Iyer PG. Screening for Barrett's esophagus and esophageal adenocarcinoma: rationale, recent progress, challenges and future directions. Clin Gastroenterol Hepatol. 2015; 13:623-634. [PubMed: 24887058]

22. Brankley SM, Halling KC, Jenkins SM, et al. Fluorescence in situ hybridization identifies high risk Barrett's patients likely to develop esophageal adenocarcinoma. Dis Esophagus. 2016; 29:513519. [PubMed: 26043762] 


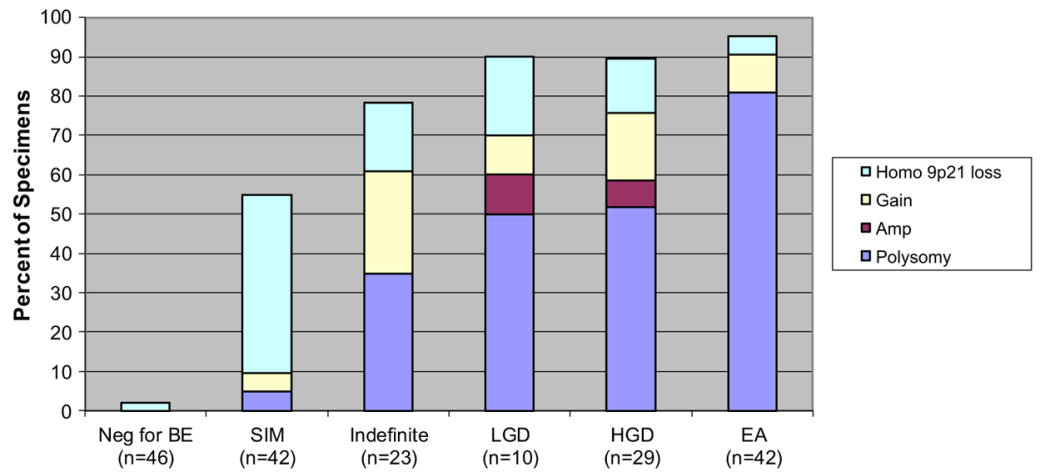

Fig. 1.

Predominant FISH abnormality per histologic classification. If more than one FISH abnormality was detected in a specimen, the most predominant (highest percentage) was used for this analysis 


\section{Table 1}

Pathologist inter-observer variability

\begin{tabular}{ll}
\hline Diagnostic criteria & Review diagnosis versus Site diagnosis (C.I.) \\
\hline EA versus rest & $.99(.97,1)$ \\
EA/HGD versus rest & $.79(.70, .87)$ \\
EA/HGD/LGD versus rest & $.73(.63, .82)$ \\
EA/HGD/LGD/IGD versus rest & $.80(.73, .88)$ \\
EA/HGD/LGD/IGD/SIM versus rest & $1.00(1,1)$ \\
\hline
\end{tabular}

Individual kappa statistics for each different histologic comparison. Each number represents the degree of inter-observer agreement between the site pathologist (Site diagnosis) and central pathologist (Review diagnosis). A kappa value of 1.00 indicates perfect agreement 


\section{Table 2}

AUC for chromosomal abnormalities

\begin{tabular}{llll}
\hline Discriminating between & \multicolumn{2}{l}{ Review diagnosis AUC } & \multirow{2}{*}{$\boldsymbol{p}$ value } \\
\cline { 2 - 3 } & \% Polysomy (C.I.) & $\boldsymbol{\%}$ Predominant 9p21 loss $\boldsymbol{a}_{\text {(C.I.) }}$ & $<.0001$ \\
\hline EA versus rest & $.84(.77, .91)$ & $.67(.59, .75)$ & .0164 \\
EA/HGD versus rest & $.83(.77, .89)$ & $.73(.66, .80)$ & .0693 \\
EA/HGD/LGD versus rest & $.83(.78, .89)$ & $.76(.69, .82)$ & .0272 \\
EA/HGD/LGD/IGD versus rest & $.84(.79, .89)$ & $.75(.67, .82)$ & $<.0001$ \\
EA/HGD/LGD/IGD/SIM versus rest & $.76(.72, .80)$ & $.90(.86, .94)$ & \\
\hline
\end{tabular}

Statistically significant values are given in bold

Comparison of different chromosomal abnormalities (polysomy vs. predominant 9p21 loss) as predictors of the degree of dysplasia. \% Polysomy has the highest AUC for all categories except that including SIM. P values are for comparison of AUCs

${ }^{a}$ Percentage of cells with 9p21 loss when 9p21 loss was the predominant FISH abnormality detected in the sample 
Table 3

Sensitivity and specificity of polysomy using overall diagnosis

\begin{tabular}{llll}
\hline Discriminating between & $\begin{array}{l}\text { AUC for \% cells with polysomy } \\
\text { (CI) }\end{array}$ & $\begin{array}{l}\text { Sensitivity of } \mathbf{1 0 \%} \text { cells with } \\
\text { polysomy (CI) }\end{array}$ & $\begin{array}{l}\text { Specificity of } \mathbf{1 0 \%} \text { cells with } \\
\text { polysomy (CI) }\end{array}$ \\
\hline EA versus rest & $.84(.77, .91)$ & $.88(.79, .97)$ & $.75(.69, .81)$ \\
EA/HGD versus rest & $.85(.79, .91)$ & $.80(.72, .88)$ & $.88(.83, .93)$ \\
EA/HGD/LGD versus rest & $.86(.80, .92)$ & $.75(.67, .83)$ & $.96(.92,1)$ \\
EA/HGD/LGD/IGD versus rest & $.85(.80, .91)$ & $.74(.66, .82)$ & $.96(.92,1)$ \\
\hline
\end{tabular}

Sensitivity and specificity using $10 \%$ polysomy (defined as $\geq 10 \%$ of cells within a sample as having polysomy) as detected by FISH. The samples analyzed were those with the highest grade diagnosis within the entire BE segment of the patient 


\section{Table 4}

The role of FISH in the detection of a field effect

\begin{tabular}{lllll}
\hline \multirow{2}{*}{ Review diagnosis } & \multicolumn{4}{l}{ Worst histology detected among other esophageal biopsies taken from the same patient (overall diagnosis) } \\
\cline { 2 - 5 } & SIM & IGD & LGD & HGD \\
\hline IGD $(\mathrm{n}=22)$ & $17 \%(1 / 6)$ & $0 \%(0 / 1)$ & $56 \%(5 / 9)$ & $100 \%(6 / 6)$ \\
LGD $(\mathrm{n}=10)$ & $100 \%(1 / 1)$ & None & $65 \%(5 / 8)$ & $100 \%(1 / 1)$ \\
\hline
\end{tabular}

Percentage of Review diagnosis samples with evidence of amplification ( $\geq 10$ signals) or polysomy based upon coincident most severe histology detected in the same esophageal segment (overall diagnosis) 